\title{
Dual-emissive, oxygen-sensing boron nanoparticles quantify oxygen consumption rate in breast cancer cells
}

\author{
Ashlyn G. Rickard, ${ }^{a}$ Meng Zhuang, ${ }^{\text {b }}$ Christopher A. DeRosa, ${ }^{\text {b }}$ \\ Xiaojie Zhang, ${ }^{a}$ Mark W. Dewhirst $\odot,{ }^{a}$ Cassandra L. Fraser, ${ }^{b}$ and \\ Gregory M. Palmer ${ }^{\mathrm{a}, *}$ \\ ${ }^{a}$ Duke University, Department of Radiation Oncology, Duke University Medical Center, \\ Durham, North Carolina, United States \\ ${ }^{\mathrm{b}}$ University of Virginia, Department of Chemistry, Charlottesville, Virginia, United States
}

\begin{abstract}
Significance: Decreasing the oxygen consumption rate (OCR) of tumor cells is a powerful method for ameliorating tumor hypoxia. However, quantifying the change in OCR is challenging in complex experimental systems.

Aim: We present a method for quantifying the OCR of two tumor cell lines using oxygensensitive dual-emissive boron nanoparticles (BNPs). We hypothesize that our BNP results are equivalent to the standard Seahorse assay.

Approach: We quantified the spectral emissions of the BNP and accounted for external oxygen diffusion to quantify OCR over $24 \mathrm{~h}$. The BNP-computed OCR of two breast cancer cell lines, E0771 and 4T07, were compared with their respective Seahorse assays. Both cell lines were also irradiated to quantify radiation-induced changes in the OCR.
\end{abstract}

Results: Using a Bland-Altman analysis, our BNPs OCR was equivalent to the standard Seahorse assay. Moreover, in an additional experiment in which we irradiated the cells at their $50 \%$ survival fraction, the BNPs were sensitive enough to quantify $24 \%$ reduction in OCR after irradiation.

Conclusions: Our results conclude that the BNPs are a viable alternative to the Seahorse assay for quantifying the OCR in cells. The Bland-Altman analysis showed that these two methods result in equivalent OCR measurements. Future studies will extend the OCR measurements to complex systems including 3D cultures and in vivo models, in which OCR measurements cannot currently be made.

(C) The Authors. Published by SPIE under a Creative Commons Attribution 4.0 Unported License. Distribution or reproduction of this work in whole or in part requires full attribution of the original publication, including its DOI. [DOI: 10.1117/1.JBO.25.11.116504]

Keywords: boron nanoparticles; oxygen imaging; oxygen consumption rate; breast cancer; dual-emission microscopy; irradiation.

Paper 200174RR received Jun. 10, 2020; accepted for publication Oct. 30, 2020; published online Nov. 23, 2020.

\section{Introduction}

The oxygen consumption rate (OCR) provides quantitative information regarding cellular metabolism and the utilization of oxygen. Oxygen is required for normal tissue function and is directly involved in the production of adenosine triphosphate (ATP) through its role in the electron transport chain. ${ }^{1}$ In cancer, a well-oxygenated tumor is more amenable to a therapeutic response, while hypoxic tumors (tumor tissue $\mathrm{pO}_{2}<10$ torr) are associated with poor therapeutic outcomes. Hypoxia develops as a result of an imbalance of oxygen supply and demand, and there is no standard method in current clinical practice to decrease hypoxia in cancer though there are a myriad of treatments (i.e., anti-angiogenetic agents) to reduce tumor hypoxia.

*Address all correspondence to Gregory M. Palmer, greg.palmer@duke.edu 
However, one possibility for ameliorating the problem of hypoxia is to decrease the OCR of tumor cells $;{ }^{2-5}$ Secomb et al, ${ }^{6}$ using a mathematical model of tumor blood vessels, predicted that a decrease of $30 \%$ in the OCR of tumor cells would abolish hypoxia; compared with increasing oxygen delivery, decreasing the OCR could be 30x more efficient. To use this therapy, a reliable, accurate method for measuring the OCR both in vitro and in vivo is necessary. Measuring OCR is complex; in vivo characterization of whole-tumor OCRs includes multiple factors that bias the data: there are strong dependences on the number of cells consuming oxygen, the type of cells, ${ }^{7}$ the presence of any mitochondrial mutations,${ }^{8-10}$ the relative tumor vascularization, ${ }^{10-12}$ and the differentiated state of the cells (non-differentiated cells are more glycolytic). ${ }^{13}$ In vivo measurements of tumor OCRs are useful in characterizing the entire tumor microenvironment, but it is nearly impossible to remove the effects of perfusion and oxygen consumption of stromal and immune cells to isolate and measure the tumor-cell OCR alone. ${ }^{14-16}$

In vitro oxygen consumption measurements are standardly performed using the Seahorse assay, which removes these in vivo variables. The Seahorse assay is used to measure the mitochondrial respiration rate by measuring the OCR before and after the addition of various inhibitors. ${ }^{17}$ The major defect in this assay is its lack of accuracy ${ }^{18}$ - the system is not airtight as it uses oxygen-permeable materials, and the diffusion of external oxygen into the cell chamber can affect measurements. ${ }^{19,20}$ Compartmental models have decreased the impact of oxygen diffusion to some extent. ${ }^{21}$ Moreover, due to the inherently large number of variables that affect OCR [plating conditions, days of growth, medium composition (both growth and assay), and concentrations of mitochondrial effectors], inter-laboratory differences have been reported, and care must be taken in the manner of presenting the data. ${ }^{22}$ In addition, there is no spatial information in Seahorse data, whereas there is potential for fluorescence-based microscopy OCR measurements to include this information.

In oncology, it is well-documented that tumor cells exhibit increased glycolysis and decreased mitochondrial activity-both drivers for changes in oxygen consumption. ${ }^{10}$ Importantly, several studies have extended these concepts to find that tumor growth can be regulated through the modulation of the OCR. ${ }^{10,16,23}$ This modulation can evoke various levels of response to standard therapies; however, radiation is of particular interest because of the extreme resistance of hypoxic tumors to radiotherapy. Several studies have successfully modulated mitochondrial activity to act as a radiosensitizer. ${ }^{24-26}$ Harnessing this knowledge for therapeutic uses requires consistent, repeatable, precise measurements of the OCR and understanding of how modulating it affects radiation therapy outcomes.

Ratiometric methods for measuring OCR in vitro have become increasingly popular due to increased resolution and real-time analysis. ${ }^{18}$ This is done through various mechanisms, usually with indirect oxygen measurements made by redox-sensitive probes or by quantifying the amount of reactive-oxygen-species. ${ }^{27-30}$ Herein, we present a novel ratiometric method for quantifying in vitro OCRs using dual-emissive, oxygen-sensing boron nanoparticles (BNPs) that directly report oxygen concentration. The BNPs are fabricated from difluoroboron $\beta$-diketonate $\left(\mathrm{BF}_{2} \mathrm{bdk}\right)$-based dye-polymer conjugates. Due to high quantum yields, large extinction coefficients, and tunable emission colors, $\mathrm{BF}_{2}$ bdk dyes have served as versatile tools for imaging and optics reagents. ${ }^{31-35}$ When the dyes are confined in a rigid polymer matrix, such as poly(lactic acid) (PLA), both fluorescence and room-temperature phosphorescence are present: the fluorescence remains unaffected by changes in oxygen concentration, but the phosphorescence is sensitive to changes in environmental oxygen via collisional quenching. ${ }^{36,37}$ The fluorescent and phosphorescent emissions are independent, with lifetimes at 462 and $569 \mathrm{~nm}$ under $\mathrm{N}_{2(\mathrm{~g})}$ to be $0.47 \mathrm{~ns}$, and $1.9 \mathrm{~ms}$, respectively. This is strong evidence that the short-lived, blue emission is fluorescence from the singlet excited-state and the long-lived, yellow emission is phosphorescence originating from the triplet-excited state. Furthermore, the yellow emission is quenched in the presence of oxygen, whereas the blue emission is unchanged according to total emission spectroscopy. While there is some spectral overlap between the two features, this is minimal. ${ }^{38}$ This unique feature makes the $\mathrm{BF}_{2}$ bdk materials useful probes in ratiometric imaging. By taking the ratio of fluorescence to phosphorescence, one can determine the concentration of oxygen in the sample while controlling for effects of particle heterogeneity, illumination intensity, and collection efficiency. In the dye-polymer conjugates, the $\mathrm{BF}_{2} \mathrm{bdk}$ 
chemical structures are related to dye properties (e.g., emission color and oxygen detection range). For example, an iodide-substituted difluoroboron dibenzoymethane poly-L-lactide or PLLA (PLA) material $\left[\mathrm{BF}_{2} \mathrm{dbm}(\mathrm{I}) \mathrm{PLA}\right]^{32}$ can sense the oxygen level from $0 \%$ to $21 \%$, while a dinapthoylmethane dye-polymer $\left[\mathrm{BF}_{2} \mathrm{dnm}(\mathrm{I}) \mathrm{PLA}\right]^{39}$ is suitable for hypoxia $(0 \%$ to $5 \%)$. The majority of physiologically based applications will occur in the $1 \%$ to $11 \% \mathrm{O}_{2}$ range. $^{40}$ We have previously demonstrated the use of these two types of BNPs in in vivo murine dorsal window chambers to measure intracellular $\mathrm{pO}_{2}$ and tumor hypoxia in the microenvironment. ${ }^{32,39}$ Recently, an iodide-substituted napthyl-phenyl derivative dye $\left[\mathrm{BF}_{2} \mathrm{nbm}(\mathrm{I}) \mathrm{PLA}\right]$ showed excellent full range oxygen detection $(0 \%$ to $100 \%)$ and was applied to monitor wound healing. ${ }^{38}$ In addition to using hydrophobic PLA as a rigid matrix, hydrophilic polymers such as poly(ethylene glycol)(PEG) are also used to create nanoparticles with improved aqueous stability. PEGylated nanoparticles also have an advantage in passive tumor targeting in which they are taken up by cells via the EPR effect. We have reported a strategy to assemble stereocomplexed PEGylated nanoparticles from boron dye-PLLA-PEG and PDLA-PEG with tumor accumulation in a murine model. ${ }^{37}$ In this study, we covalently linked the full range boron dye to PLLA-PEG and applied the established PEGylation method to generate oxygen-sensing BNPs for measuring OCR. ${ }^{37}$

Our method fills an important gap in metabolic measurements because, while standard Seahorse techniques can be expanded from 2D monolayers of cells to ex vivo 3D tissues, they rely on inducing changes in oxygen consumption by inhibitors of mitochondrial function. Our proposed method translates from standard 2D cultures to 3D organs. We have previously used BNPs for straightforward $\% \mathrm{O}_{2}$ imaging in in vivo murine dorsal window chamber models that accommodate the depth limitations of optical wavelengths. ${ }^{33,38}$ Our objective here is to measure and compare the in vitro OCR measured in two breast cancer cell lines, E0771 and 4T07, with the gold-standard Seahorse assay. This is the first step toward our ultimate goal of extending these OCR measurements in vivo. To measure the OCR, BNPs are added to cell media (or any other experimental solution) and their phosphorescence and fluorescence intensity is measured in a spectroscopic plate reader. In our study, we also irradiated each cell line to reach $50 \%$ survival to determine if the basal OCR is changed. We hypothesize that (1) OCRs of E0771 and $4 \mathrm{~T} 07$ can be quantified via ratiometric sensing with the BNP, (2) OCRs measured via the plate reader are comparable to the gold-standard Seahorse assay, and (3) OCR is reduced after a dose of radiation that reduces survival by $50 \%$.

\section{Experimental Methods and Materials}

\subsection{BNP Synthesis Materials and Methods}

The lactide monomer (D-lactide) was a generous gift from Corbion Purac $(\mathcal{C}$. L-Lactide was purchased from TCI America. Lactide was recrystallized twice from EtOAc and dried in vacuo overnight prior to use. PEG was purchased from Sigma Aldrich (2 $000 \mathrm{Da}, \mathrm{Ð}=\sim 1.05$ ) and dried via azeotropic distillation in toluene according to a previously described protocol. ${ }^{41}$ The PEG was stored under nitrogen in a glovebox prior to use as a macroinitiator. The polymers PEG-PLLA-OH (GPC: $M_{\mathrm{n}}=6900 \mathrm{Ð}=1.04$, and ${ }^{1} \mathrm{HNMR}=7000$ ) and PEG-PDLA-OH $\left(M_{\mathrm{n}}=12500, \mathrm{Ð}=1.08 \text {, and }{ }^{1} \mathrm{HNMR}=13800\right)^{36}$ and the ester, 6-iodo, 2-methylnaphthoate ${ }^{42}$ were prepared as previously described. ${ }^{1} \mathrm{H}$ NMR spectra are in accord with literature values. Solvents $\mathrm{CH}_{2} \mathrm{Cl}_{2}$ and THF were dried over $3 \AA$ molecular sieves activated at $300^{\circ} \mathrm{C}$, transferred via cannula, and dried a second time over $3 \AA$ molecular sieves activated at $300^{\circ} \mathrm{C} .{ }^{43}$ The solvents were stored in a dry pot. All other chemicals were reagent grade from Sigma-Aldrich and were used without further purification.

Synthetic Methods ${ }^{1} \mathrm{H}$ NMR spectra $(600 \mathrm{MHz})$ were recorded on a Varian VMRS 600/51 instrument in $\mathrm{CDCl}_{3}$ or $\mathrm{D}_{6}$-DMSO. ${ }^{1} \mathrm{H}$ NMR spectra were referenced to the residual signals for protiochloroform $(7.26 \mathrm{ppm})$, protioDMSO $(2.50 \mathrm{ppm})$, and protioacetone $(2.09 \mathrm{ppm})$. In the ${ }^{1} \mathrm{H}$ NMR assignments, aromatic positions are defined for phenyl $(\mathrm{Ph})$ and naphthyl $(\mathrm{Np})$ positions. Coupling constants are given in hertz. Number average molecular weights $\left(M_{n}\right)$, weight average molecular weights $\left(\mathrm{M}_{\mathrm{w}}\right)$, and polydispersity index $(Ð)$ were determined by 

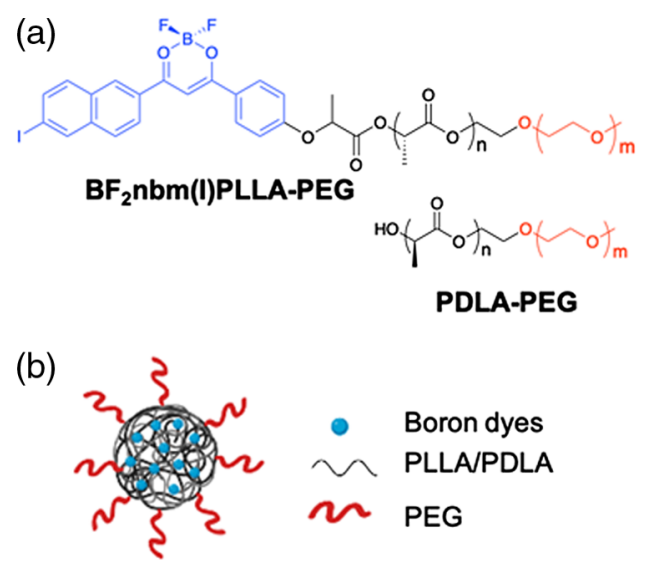

Fig. 1 Nanoparticle design and composition of stereocomplex nanoparticles: (a) chemical structures of BF2nbm(I)PLLA-PEG and PDLA-PEG polymers. (b) Schematic illustration of stereocomplex PEGylated nanoparticles.

gel permeation chromatography $(\mathrm{GPC})\left(\mathrm{THF}, 25^{\circ} \mathrm{C}, 1 \mathrm{ml} / \mathrm{min}\right)$ using multiangle laser light scattering $\left(\lambda=658 \mathrm{~nm}, 25^{\circ} \mathrm{C}\right)$ and refractive index $\left(\lambda=658 \mathrm{~nm}, 25^{\circ} \mathrm{C}\right)$ detection. A Polymer Laboratories $5 \mu \mathrm{m}$ mixed-C guard column and two GPC columns along with Wyatt Technology Corp. (Optilab REX interferometric refractometer, miniDawn TREOS laser photometer) and Agilent Technologies instrumentation (series 1260 HPLC) and Wyatt Technology software (ASTRA 6.0) were used for analysis. The incremental refractive index $(d n / d c)$ was determined by a single-injection method assuming $100 \%$ mass recovery from the columns. UV-vis spectra were recorded on a Hewlett-Packard 8452A diode-array spectrophotometer.

Figure 1 shows the stereocomplex nanoparticle design and composition.

\subsection{Polymer Synthesis}

$n b m(I) O H$ : The iodo-substituted, naphthyl-phenyl ligand was prepared as previously described by Jin et al, ${ }^{44}$ for 3-hydroxy-1-(4-hydroxyphenyl)-3-phenylprop-2-en-1-one, except the aromatic ester 6-iodo, 2-methylnaphthoate was used in place of methyl benzoate to yield a dark brown powder; $178 \mathrm{mg}(13 \%) .{ }^{1} \mathrm{H}$ NMR: $\left(600 \mathrm{MHz}, \mathrm{D}_{6}\right.$-DMSO) $\delta 17.38(\mathrm{~s}, 1 \mathrm{H}$, enol-OH), 10.47 (s, broad, $1 \mathrm{H}$, phenol-OH), $8.75(s, 1 \mathrm{H}, 1-\mathrm{NpH}), 8.48(s, 1 \mathrm{H}, 5-\mathrm{NpH}) 8.18(d$, $J=12,3-\mathrm{NpH}), 8.08(d, J=12,2 \mathrm{H}, 2,6-\mathrm{PhH}), 7.98(d, J=12,1 \mathrm{H}, 4-\mathrm{NpH}) 7.85$ ( $s$, broad, $2 \mathrm{H}, 7,8-\mathrm{NpH}), 7.34$ ( $s, 1 \mathrm{H}, \mathrm{COCHCO}), 6.90(d, J=12,2 \mathrm{H}, 3,5-\mathrm{PhH})$ (Fig. S2). HRMS [electrospray ionization (ESI), time-of-flight (TOF)] $\mathrm{m} / \mathrm{z}$ calcd for $\mathrm{C}_{19} \mathrm{H}_{12} \mathrm{O}_{3} \mathrm{I}: 414.9831$ $[\mathrm{M}-\mathrm{H}]^{+}$; found 414.9825 .

$\mathrm{BF}_{2} \mathrm{nbm}(\mathrm{I}) \mathrm{OH}$ : The boron dye coupler was prepared as previously described ${ }^{37}$ but with $\mathrm{nbm}(\mathrm{I}) \mathrm{OH}$ to yield a yellow powder after recrystallization from acetone/hexanes; $45 \mathrm{mg}(35 \%)$. 1H NMR: (600 MHz, D 6 -DMSO) $\delta 11.22$ (s, broad, $1 \mathrm{H}$, phenol-OH), 8.99 (s, $1 \mathrm{H}, 1-\mathrm{NpH}), 8.55$ (s, $1 \mathrm{H}, 5-\mathrm{NpH}), 8.33(\mathrm{~m}, 3 \mathrm{H}, 2,6-\mathrm{Ph} H, 3-\mathrm{NpH}), 8.07(d, J=6,1 \mathrm{H}, 4-\mathrm{NpH}), 7.95(\mathrm{~m}, 2 \mathrm{H}, 7$, 8-NpH), 7.87 (s, 1H, COCHCO), $7.00(d, J=6,2 \mathrm{H}, 3,5-\mathrm{PhH})$ (Fig. S3). HRMS (ESI, TOF) $\mathrm{m} / \mathrm{z}$ calcd for $\mathrm{C}_{19} \mathrm{H}_{11} \mathrm{BO}_{3} \mathrm{~F}_{2} \mathrm{I}: 462.9814[\mathrm{M}-\mathrm{H}]^{+}$; found 462.9814 .

$B F_{2} n b m(I) P L L A-P E G$ : The dye-coupled polymer was prepared as previously described by Kerr et $\mathrm{al}^{37}{ }^{37}$ except the dye, $\mathrm{BF}_{2} \mathrm{nbm}(\mathrm{I}) \mathrm{OH}$, was used in placed of $\mathrm{BF}_{2} \mathrm{dbmOH}$. A lower MW mPEG-PLLA-OH block copolymer (PEG $=2000 \mathrm{Da}$ and PLLA $=5000 \mathrm{Da})$ was also used in this reaction, prepared as previously described. ${ }^{2}$ The product was obtained as a yellow powder; $513 \mathrm{mg}(76 \%) \cdot M_{\mathrm{n}}(\mathrm{GPC} / \mathrm{MALS})=7600(\mathrm{~d} n / \mathrm{d} c=0.056), \mathrm{D}=1.05\left({ }^{1} \mathrm{HNMR}\right)=7300 ;{ }^{1} \mathrm{H}$ NMR: $\left(600 \mathrm{MHz}, \mathrm{CDCl}_{3}\right) \delta 8.69(\mathrm{~s}, 1 \mathrm{H}, 1-\mathrm{NpH}), 8.32(s, 1 \mathrm{H}, 5-\mathrm{NpH}), 8.17(d, J=6,2 \mathrm{H}, 2$, $6-\mathrm{PhH}), 8.09(d, J=12,1 \mathrm{H}, 3-\mathrm{NpH}), 7.85(m, 2 \mathrm{H}, 7,8-\mathrm{NpH}), 7.72(d, J=12,1 \mathrm{H}, 4-\mathrm{NpH})$, $7.21(s, 1 \mathrm{H}, \mathrm{COCHCO}), 7.06(\mathrm{~d}, J=6,2 \mathrm{H}, 3,5-\mathrm{PhH}), 5.17(q, J=6,66 \mathrm{H}$, PLLA-H $)$, $3.62\left(s\right.$, broad, $179 \mathrm{H}$, PEG-OCH $\left.{ }_{2} \mathrm{CH}_{2}-\right), 3.36\left(s, 3 \mathrm{H}, \mathrm{PEG}-\mathrm{OCH}_{3}\right), 1.55(m$, broad, $217 \mathrm{H}$, PLLA-CH ${ }_{3}$ ) (Fig. S4). 


\subsection{Nanoparticle Fabrication}

Stereocomplex nanoparticles, as shown in Fig. 1, were fabricated as previously described. ${ }^{37}$ In brief, oxygen-sensing polymer $\left(\mathrm{BF}_{2} \mathrm{nbm}(\mathrm{I}) \mathrm{PLLA}-\mathrm{PEG}\right)(45 \mathrm{mg})$ and mPEG-PDLA $(45 \mathrm{mg})$ were dissolved in DMF $(9 \mathrm{ml})$. The solution was briefly heated to facilitate dissolution of the polymers. With a syringe pump, the DMF solution was added to deionized (DI) water (81 ml) at a constant rate of $1 \mathrm{ml} / \mathrm{min}$. The solution was filtered through Whatman filter paper, and DMF was removed by dialysis (Select/Por; 12 to $14 \mathrm{kDa}$ molecular weight cutoff; $2-1$ beaker filled with DI water; water changed every $2 \mathrm{~h}$ for $6 \mathrm{~h}$, then allowed to stand overnight). The nanoparticle solution was then passed through a Whatman 200-nm Anotop filter. Nanoparticle sizes and polydispersities were analyzed via dynamic light scattering (DLS, Wyatt, DynaPro Plate Reader II), and results were consistent with previous reports (Fig. S5). ${ }^{25}$

\subsection{Cell Culture}

The murine breast cancer cell line E0771 (CH3 Biosystems, Amherst, New York) was cultured in RPMI $1 \times 1640$ with $300 \mathrm{mg} / \mathrm{L}$ of L-glutamine (Gibco Gaithersburg, Maryland). 4T07 cells were acquired from American Type Culture Collection and cultured in DMEM with $4.5 \mathrm{~g} / \mathrm{L}$ of D-glucose, $584 \mathrm{mg} / \mathrm{L}$ of L-glutamine, and $110 \mathrm{mg} / \mathrm{L}$ sodium pyruvate (Gibco Gaithersburg, Maryland). Both cell media were supplemented with $10 \% \mathrm{v} / \mathrm{v}$ heat-inactivated fetal bovine serum (HI-FBS) (Gibco Gaithersburg, Maryland) and 1\% v/v anti-mycotic/antibiotic (A/A) (Gibco, Gaithersburg, Maryland). Cells were maintained in a humidified $37^{\circ} \mathrm{C}$ incubator with $5 \% \mathrm{CO}_{2}$. Prior to the experiment, two black, 96-well plates with clear bottoms (Greiner, Bio-One, Kremsmünster, Austria) per cell line were plated with $4 \times 10^{4}$ cells/well and incubated for $24 \mathrm{~h}$. This resulted in near 100\% confluence for each cell line at the time of the study.

\subsection{Boron Nanoparticle Calibration}

To quantify oxygen concentrations in experimental conditions, BNPs were calibrated with a spectrometer (SpectraMax M5, Molecular Devices, San Jose, California). A solution of 1:5 v/v BNP:deionized water was placed in a clear cuvette, and spectra were measured for $0 \% \mathrm{O}_{2}, 5 \% \mathrm{O}_{2}, 10 \% \mathrm{O}_{2}, 15 \% \mathrm{O}_{2}, 20 \% \mathrm{O}_{2}, 50 \% \mathrm{O}_{2}$, and $100 \% \mathrm{O}_{2}$. Oxygen gas was mixed with nitrogen gas to make these defined oxygen concentrations. These gases were bubbled into test solutions for $5 \mathrm{~min}$ prior to data acquisition. The top of the cuvette was covered with two layers of Parafilm (American National Can, Menasha, Wisconsin) to minimize oxygen diffusion into the cuvette from the open end. A GFC mass flow controller (Aalborg, Orangeburg, New York) with an oxygen sensor ( $\mathrm{MaxO}_{2}$, Ceramatec Inc., Salt Lake City, Utah) in series with the gas line was used to change the molecular oxygen and nitrogen mixture and to ensure accuracy, respectively. The solution was calibrated at $37^{\circ} \mathrm{C}$. The solution temperature was measured with a thermocouple (Model HH23, OMEGA Engineering, Inc., Norwalk, CT). The excitation wavelength was $405 \mathrm{~nm}$. The emission spectrum ( $450 \mathrm{~nm}$ to $600 \mathrm{~nm}$ ) was measured at $10 \mathrm{~nm}$ step increments [Fig. 2(B)]. This spectrometer system was also used for data acquisition during experiments.

In MATLAB (v R2018b, Mathworks, Natick, Massachusetts), spectrometer data were used for a parallel factor analysis (parafac.m function in N-Way Toolbox ${ }^{45}$ ) that computes two non-negative intensity components that contribute to each wavelength measurement. These two components are the fluorescence and phosphorescence and are most clearly seen in Fig. 2(C) at $0 \% \mathrm{O}_{2}$. The component spectra for all oxygen percentages are shown in Fig. 2(E). By breaking down the spectral components of each wavelength and each percent $\mathrm{O}_{2}$, the proceeding fluorescent to phosphorescent $(\mathrm{F} / \mathrm{P})$ ratio was computed more accurately by determining the exact percentage that each component contributes to the total signal. By repeating this spectral decomposition for each known oxygen concentration, a calibration curve and resulting quadratic fit [Eq. (1), Fig. 2(d)] were determined. This calibration curve was used for the remaining experiments to calculate the percent $\mathrm{O}_{2}$ from the measured F/P ratio [Fig. 2(c)]. It was found that a quadratic fit the data well $\left(R^{2}=0.9985\right)$ and the expected standard error of predictions was $<0.6$; thus we can use the straightforward relationship shown below: 

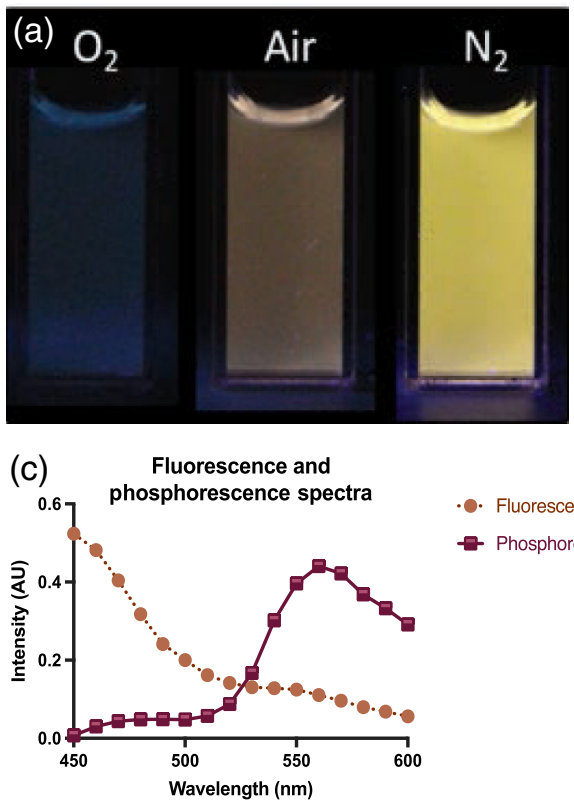

(b)

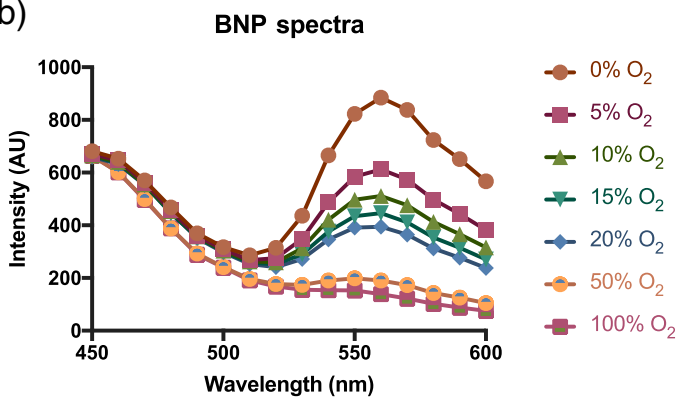

(d)

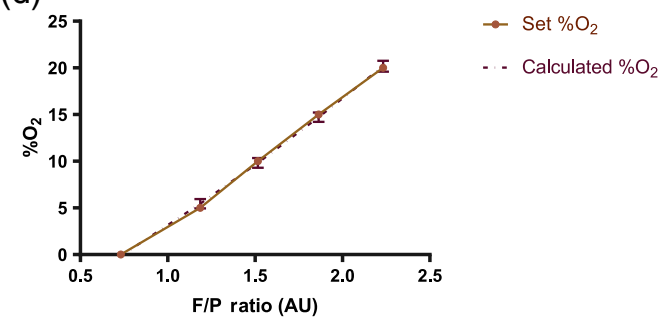

(e) Separated components for F and P

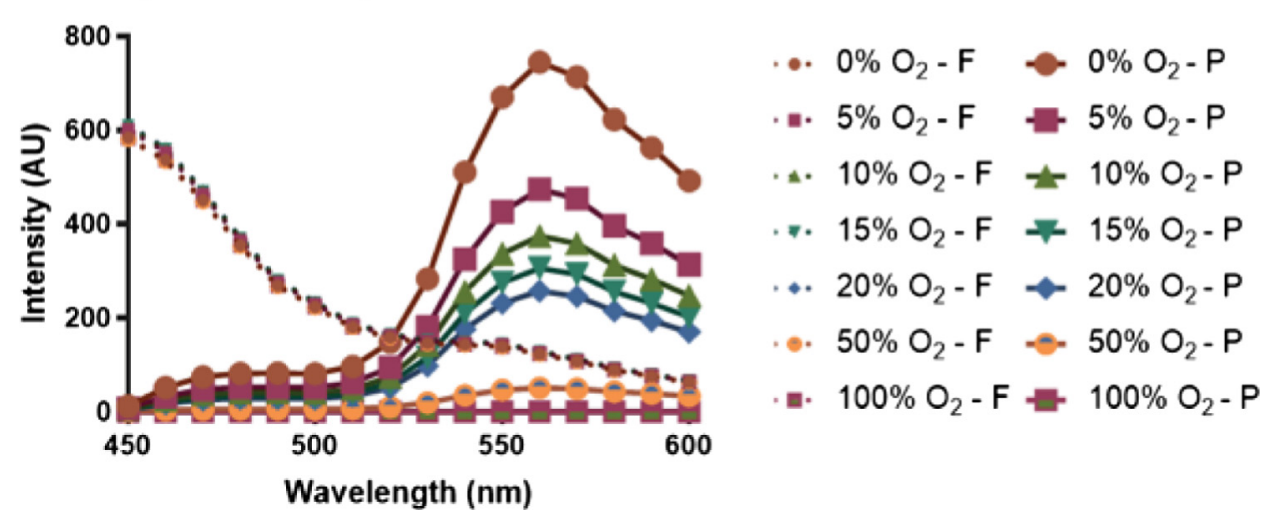

Fig. 2 Procedure for BNP Calibration. (a) BNPs in the presence of $100 \% \mathrm{O}_{2}$ (blue), room air (brown), and $100 \% \mathrm{~N}_{2}$ (yellow) show the color difference as the emission changes in various environments. (b) Raw BNP spectra in the presence of $0 \%, 5 \%, 10 \%, 15 \%, 20 \%, 50 \%$, and $100 \% \mathrm{O}_{2}$. The fluorescence and the phosphorescence are independent; the fluorescence remains steady at $450 \mathrm{~nm}$, and the phosphorescence decreases with increasing oxygen percentage around $560 \mathrm{~nm}$. (c) By decomposing the full BNP spectra $\left(\right.$ at $\left.5 \% \mathrm{O}_{2}\right)$ into its separate fluorescent and phosphorescent components that make up the entire signal, the contamination of the independent spectra can be taken into account. (d) The calibration curve shows that the F/P (fluorescence to phosphorescence) ratio against oxygen percentages is quadratic over the entire curve $\left(0 \%\right.$ to $\left.100 \% \mathrm{O}_{2}\right)$. Because our sample will be in the $0 \%$ to $21 \%$ oxygen percentage range and the BNPs are most sensitive at low oxygenations, our fit is shown from $0 \%$ to $20 \%$ oxygen with standard error bars representing the expected error estimate-based predictions, representing the range of possible $\mathrm{O}_{2}$ values, around the fit. (e) The fully decomposed fluorescent and phosphorescent spectra are shown. The solid lines represent fluorescence $(F)$ and the dotted lines represent phosphorescence $(P)$.

$$
\text { Percent Oxygen }=1.12\left(\frac{F}{P}\right)^{2}+10.23\left(\frac{F}{P}\right)-8.25
$$

\subsection{Seahorse Assay}

We used previously published work examining oxygen consumption by $4 \mathrm{~T} 07$ versus E0771 cells via the Seahorse metabolic assay as shown in Ref. 17. In that study, the XF24 Analyzer from Seahorse Biosciences was used to measure the bioenergic profile of 4T07 and E0771 mammary 
carcinoma cell lines, which were plated in culture to confluency. Real-time measurements of OCR, extracellular acidification rate, and proton production rate were obtained. These mitochondrial function parameters were determined in the presence of mitochondrial uncoupling agents: oligomycin, FCCP (trifluoromethoxy carbonylcyanide phenylhydrazone, an uncoupler that disrupts ATP by moving protons across cell membranes), and antimycin. Of note, these agents were injected sequentially through ports in the XF Assay cartridges to final concentrations of $1 \mu \mathrm{g} / \mathrm{ml}, 1 \mu \mathrm{M}$, and $10 \mu \mathrm{M}$, respectively. The addition of oligomycin, an ATPase inhibitor, shows OCR associated with ATP production. The maximal respiratory rate as reflected by oxygen consumption is determined by the FCCP injection, which transfers $\mathrm{H}^{+}$across the cell membrane prior to use for oxidative phosphorylation. Finally, the addition of rotenone, which inhibits complex I, halts mitochondrial respiration. The study allowed for the determination of baseline mitochondrial activity, OCR from ATP production, and maximal respiration.

\subsection{Experimental Setup for BNP OCR Assessment}

The 96-well black plates were setup with twelve wells for each experimental condition, six wells with cells and six wells without cells as shown in Table S1 (Supplemental Material). The volume of BNP solutions covering the wells was $50 \mu \mathrm{l}$. The volume of a mineral oil barrier that slowed the external oxygen diffusion into the system during imaging was $100 \mu \mathrm{l}$ (Fig. 3). Each solution consisted of $20 \% \mathrm{v} / \mathrm{v}$ BNPs. The controls for the experiment were four wells of BNPs, BNPs with mineral oil, and mineral oil alone. The experimental conditions include the following solutions: BNPs and cell media with cells; BNPs and cell media without cells; and BNPs, cell media, and $20 \mathrm{mM}$ of sodium dithionite without cells. The latter, an oxygen scavenger, acted as a positive control. All experimental cell media were supplemented with $10 \% \mathrm{v} / \mathrm{v}$ HI-FBS, $1 \% \mathrm{v} / \mathrm{v}$ A/A and were phenol-red free media (either RPMI or DMEM) (Gibco Gaithersburg, MD).

\subsection{Irradiation of Cells}

An x-ray irradiator (X-RAD 320, Precision X-Ray, North Branford, CT) was used for the irradiation portion of each study. During transport, cells were covered and remained at approximately $37^{\circ} \mathrm{C}$ via a paraffin wax pad. Each cell line was irradiated at its $50 \%$ survival dose: 1.85 Gy for E0771 and 2.49 Gy for 4T07, according to clonogenic survival assays. The cells were irradiated at a dose rate of $1.96 \pm 0.54 \mathrm{~Gy} / \mathrm{min}$ with a standard F1 filter, which corresponds to 2-mm aluminum beam hardening. The voltage was at $320 \mathrm{kVp}$, and the current was $10 \mathrm{~mA}$. These values also approximate fractionated radiation doses used clinically.

\subsection{Experimental Spectral Acquisition}

The plate spectra were measured as described above for the calibration. Immediately after irradiation, the wells were aspirated and experimental solutions were added. Cells were continuously kept at $37^{\circ} \mathrm{C}$ during these experiments. Spectra were measured at 0-, 1-, 2-, 4-, 6-, and 24-h post-irradiation. Measurement time points were rounded to the nearest hour, and cells were incubated and stored in a light-free environment between each acquisition.

\subsection{Microscope Imaging}

The BNPs can be visualized in a standard confocal microscope (Zeiss Axio Observer.Z1, Carl Zeiss Microscopy, Cambridge, Massachusetts) with the addition of a Quad-View system (QV2, Photometrics, Tucson, Arizona), which uses a series of beamsplitters and bandpass filters to split light from the sample onto four quadrants of the detection camera (Orca Flash, Hamamatsu, $2048 \times 2048$ pixels, Bridgewater, New Jersey). BNP measurements involved a filter set designed to detect light at 470, 500, 535, and $560 \mathrm{~nm}$ with a passband of $\sim 30 \mathrm{~nm}$ (Brightline Fluorescence Filters, 560/25 nm, and 500/24 nm, Semrock) and a mercury lamp (X-CITE 120 Lamp, Lumen Dynamics, Mississauga, Ontario). A long-pass fluorescence filter was used to illuminate the sample with excitation light at $430 \mathrm{~nm}$, while the fluorescence and phosphorescence at higher wavelengths were passed through to the detection optics. A background image taken from a cell 


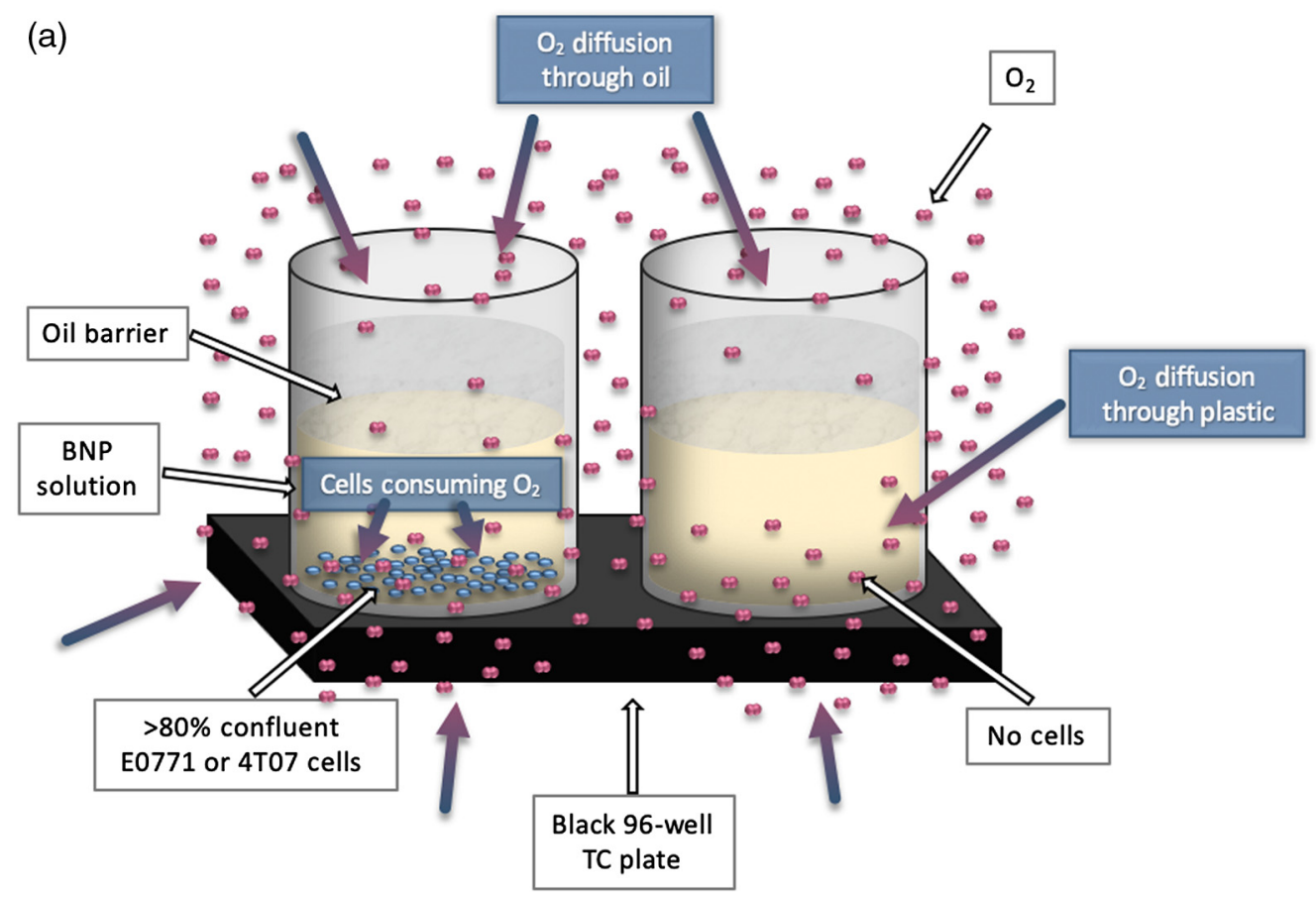

(b)

E0771

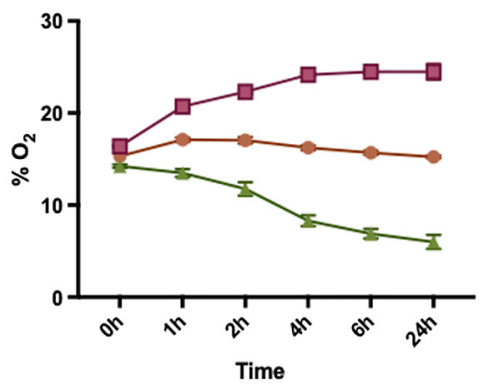

4T07

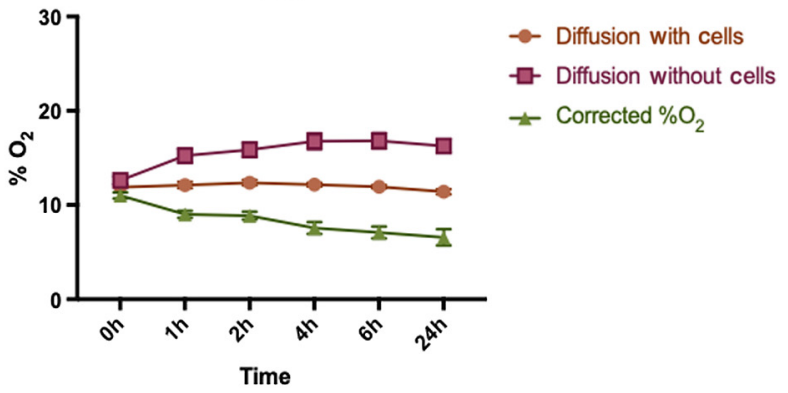

Fig. 3 Diffusion and corrected oxygen data for E0771 and 4T07: (a) Despite the oil barrier that was placed on top of the experimental solution (both with cells or without), there is significant external oxygen diffusing into the wells. For the two cells lines E0771 and 4T07, we corrected for the diffusion by measuring the $\% \mathrm{O}_{2}$ with and without cells. (b) The difference between these measurements results in the actual $\% \mathrm{O}_{2}$ in the solution with cells. This is the first step in calculating the OCR.

plate well that contained only the media solution was imaged and was subtracted from the acquired BNP image in MATLAB. The QV2 image was aligned as before by cropping each individual wavelength image and then registering each image with an affine transformation to the 500-nm image. The ratio of the intensity detected from the emission at 565 and 500-nm $(\mathrm{F} / \mathrm{P})$ of the BNPs was computed over each image pixel. Because the optics of this system are unlike the plate reader, a microscope-specific calibration curve was obtained in the same manner as described in Sec. 2.4. For each time point (except 24-h post-irradiation), the F/P ratio was microscopically imaged post-plate-reader acquisition. Once the average F/P ratio was obtained over each image, the data were analyzed in the same manner as the spectra.

Immediately after the spectra were obtained with the plate reader, a microscopic image was taken of the same plate before it was placed back in the incubator.

\subsection{Spectral Analysis and Oxygen Consumption Rate Calculation}

As previously described in the BNP calibration, a spectral decomposition in MATLAB was performed for the entire plate. From this, the full fluorescence and phosphorescence spectra 
were attained, and the F/P ratio was calculated. Then, using the calibration curve, the percent oxygen was quantified for each well. The control groups (BNPs, BNPs with mineral oil, mineral oil alone) were measured: the BNPs reported the expected spectra at $21 \% \mathrm{O}_{2}$, and the mineral oil did not contribute any significant fluorescence. The BNPs were confirmed to report the expected spectra in a low-oxygen environment via the BNPs + sodium dithionite group, where the sodium dithionite scavenged $\mathrm{O}_{2}$ within the well. The remainder of the analysis is in the experimental group: BNPs + cell media. Our first task was to correct for external diffusion [Fig. 3(a)]. The diffusion rate of air into the plate was quantified using the wells without the cells by calculating the rate of change of oxygen. We plotted this diffusion rate against the oxygen values (still using wells without cells) to obtain a curve and fit that accounts for the amount of oxygen in the well via external diffusion. Similarly, the change in oxygen over time was quantified in the group that contained cells; diffusion was corrected for using the equation of the fit described above [Fig. 3(b)]. Finally, the OCR was calculated using the change in oxygen percentage over time, corrected for diffusion effects [Eq. (2)]:

$$
\operatorname{OCR}\left(\% \mathrm{O}_{2}, t\right)=\frac{\Delta \% \mathrm{O}_{2}}{\Delta t}-\mathrm{D}\left(\% \mathrm{O}_{2}\right)
$$

where $D$ is diffusion and $t$ is time.

\subsection{Statistical Analysis}

A Bland-Altman analysis was performed to compare the Seahorse and BNP assays. A two-way analysis of variance (ANOVA) was applied to determine if irradiation significantly altered the basal OCR (versus the unirradiated control) followed by Tukey's post-hoc test. A $p<0.05$ was considered significant.

\section{Results}

\subsection{Seahorse Assay and BNP Equivalence}

Figure 4 compares the Seahorse assay results with the BNP OCR results. Figures 4(a) and 4(b) plot the mean Seahorse data (prior to exposure of any external agents) on the lower $x$ axis, while the mean BNP data are plotted on the upper $x$ axis. The methods appear relatively consistent with each other for both cell lines. Figures 4(c) and 4(d) show the results of a Bland-Altman analysis for each cell line. The difference of the methods versus the average is plotted with the $95 \%$ confidence intervals shown in dotted lines. The data points for both cell lines show a scattering above and below the $x$ axis and within the confidence intervals. This shows that the two methods for measuring and quantifying the OCR are equivalent.

\subsection{Irradiated versus Unirradiated Cells}

The OCR, with and without irradiation, is shown in Fig. 5 for E0771 and 4T07. The full experimental data can be found in the Supplemental Material (Figs. S8-S10), including the F/P ratios and percent oxygenation. The trends are similar in both cell lines, increasing over time, after receiving their 50\% survival fraction (SF) dose. For E0771 [Fig. 5(A)], the unirradiated OCR is consistently larger than the irradiated group. A two-way ANOVA reports significance for each time point $(p<0.0001)$. Conversely, for 4 T07 [Fig. 5(B)], the irradiated OCR is larger than the unirradiated and only the first timepoint shows a significant $(p=0.0255)$ difference. Table S2 contains the ANOVA tables in the Supplemental Material.

\subsection{OCR via Optical Microscopy}

Figure 6 shows the results of the microscope data. Unlike the plate reader, which is a monochromator, the microscope uses filters that are centered at the fluorescence and phosphorescence emissions. Because of these specific optics and the camera (which may be more sensitive to 

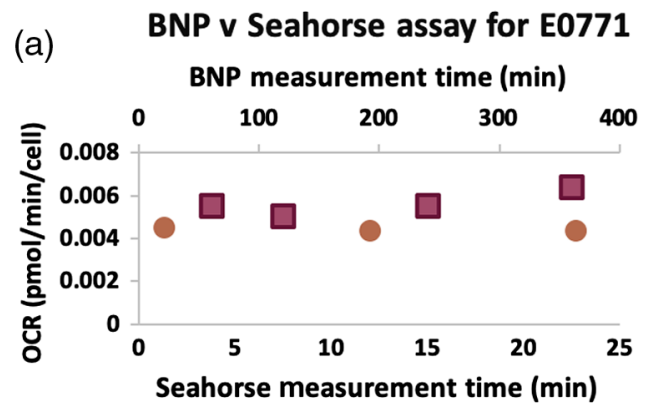

Seahorse Assay: E0771 口BNP: E0771

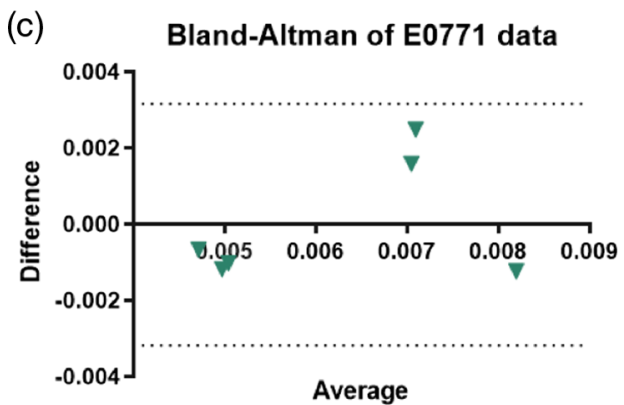

(b)

BNP v Seahorse assay for $4 \mathrm{T07}$

BNP measurement time (min)

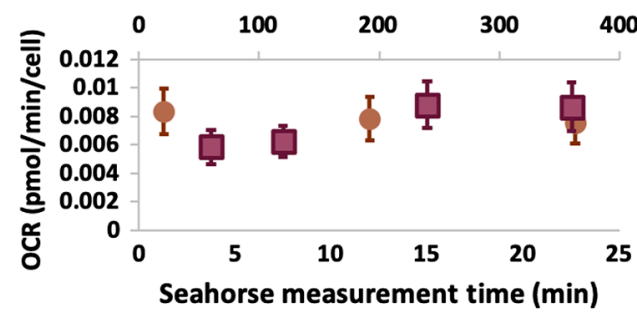

Seahorse Assay: 4T07 口BNP: 4 TT07

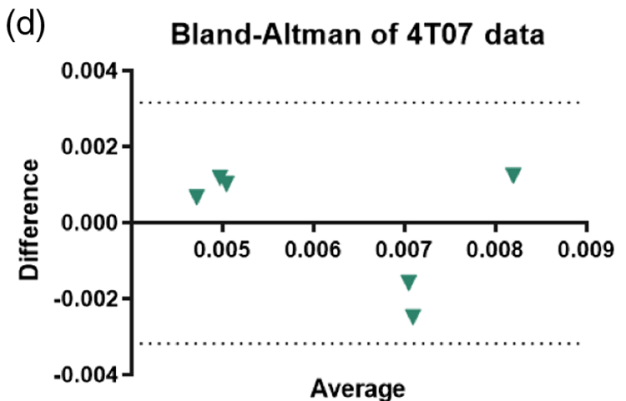

Fig. 4 Equivalence of BNP versus Seahorse assay for OCR measurements: For both E0771 (a) and 4T07 (b), we compare the gold-standard Seahorse assay OCR with the BNP OCR measurements. Because of the different measurement time points, 0 to $30 \mathrm{~min}$ for the Seahorse assay and 60 to $360 \mathrm{~min}$ for the BNPs, there are two horizontal axes. For each cell line, the Seahorse and BNP data are remarkably similar with the mean of six wells approaching the OCR value measured with the Seahorse assay. The error bars are the standard error of the mean, and some error bars are too small to be resolved with the symbol. (c) and (d) The Bland-Altman plots in which the difference between the BNP and Seahorse measurements are plotted against their average. Because the data points are within the dotted $95 \%$ confidence interval and are scattered on either side of the $x$ axis, the two methods are considered unbiased and comparative.

(a)

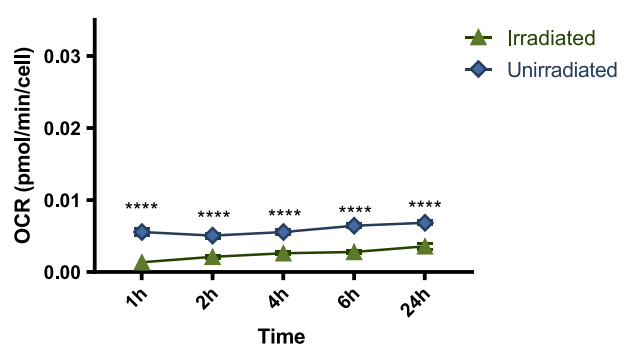

(b) OCR: 4T07

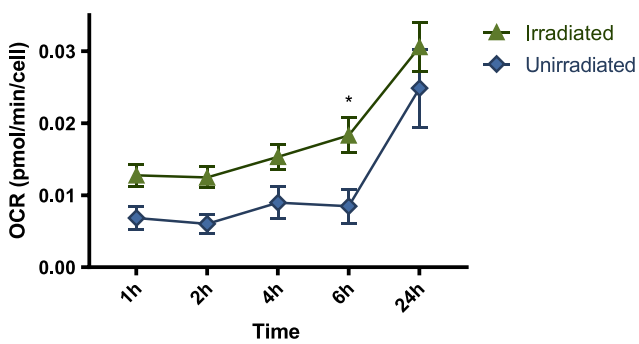

Fig. 5 Irradiated and unirradiated OCR for E0771 and 4T07: (a) The irradiated and unirradiated OCR for E0771 and (b) the irradiated and unirradiated OCR for 4T07. Both cell lines were irradiated with their $50 \%$ SF, and unirradiated cells were sham irradiated. The error bars represent the standard error of the mean. Notably, both cells lines demonstrate a difference between those exposed and those unexposed to radiation, with E0771 demonstrating a significant difference at all timepoints. Significant $p$-values of $<0.0001$ are represented by ${ }^{* \star * *}$ for E0771, and ${ }^{*}$ for 4T07 is $p=0.0479$ via a two-way ANOVA and Tukey's multiple comparisons test.

one end of the spectrum), we calibrated the BNPs in this system by taking images of different percent $\mathrm{O}_{2}$ and calibrating it against the F/P ratio as described in Sec. 2.4. In Fig. 6(a), the images show the fluorescence and phosphorescence images for each cell line. Recall that these images are obtained via a QuadView system that splits the image into four quadrants, two of which are 
(a)
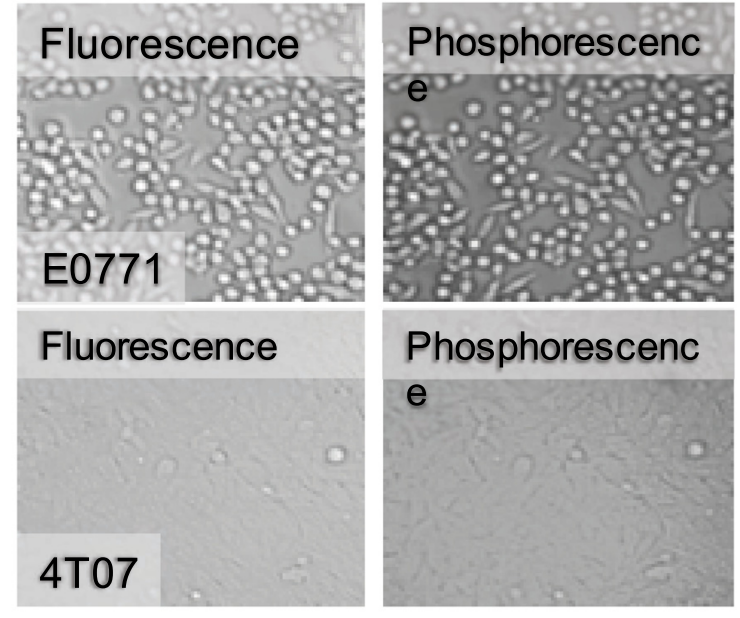

(b)

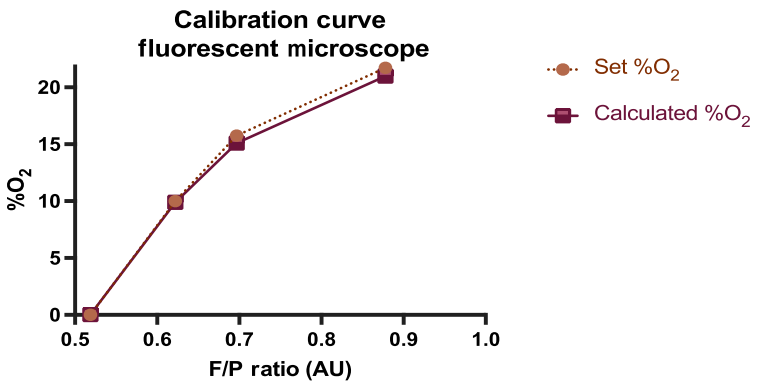

(c)

OCR: E0771

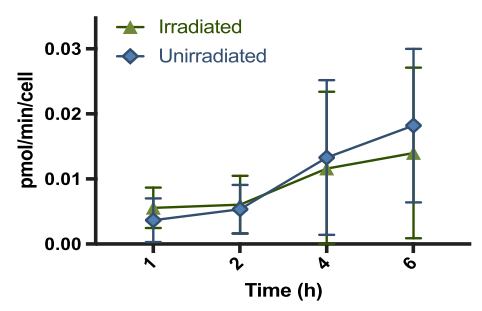

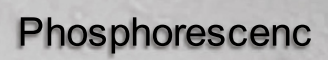

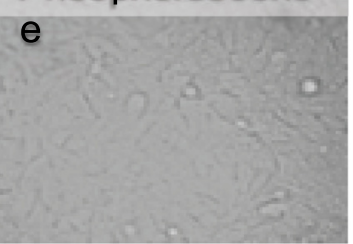

OCR: 4T07

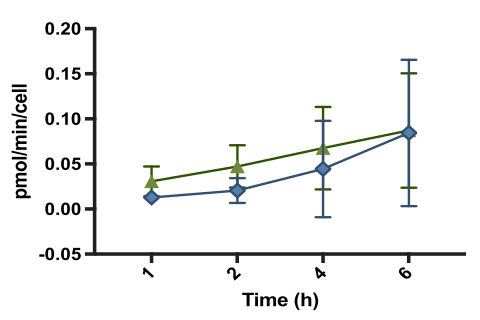

Fig. 6 OCR data acquired on microscope system: (a) microscope images of fluorescence and phosphorescence for each cell line using the QuadView filter system. It is worth noting that the microscope does not have a phase-contrast protocol, so the cells are difficult to visualize. (b) The BNP calibration on the microscope shows a quadratic relationship between the F/P ratio and percent $\mathrm{O}_{2}$. (c) The OCR of E0771 and 4T07 as calculated from the microscope data is much noisier than the plate reader; however, it should be noted that the analysis averages the entire image not just the area of the cells. The standard deviation bars are quite large, and there is no significance between irradiated versus unirradiated groups. The 4T07 group, compared with the plate reader data, shows the same trend, with the irradiated group having a larger OCR compared with unirradiated.

centered around 500 (fluorescence) and $564 \mathrm{~nm}$ (phosphorescence). The microscope does not have phase-contrast, so the cells are difficult to visualize. Of the easily-visualized cells, the rounded, unhealthy cells (notable in E0771 images) are the most obvious. We did not measure the apoptotic fraction during this experiment, but it is clearly important as fewer cells will lead to a naturally decreased OCR. The calibration [Fig. 6(b)] shows a quadratic relationship between the F/P ratio and the percent oxygen. Figure 6(c) shows the mean OCR results for E0771 and 4T07, comparing irradiated and unirradiated groups. The standard deviation error bars are much larger in the microscope dataset compared with the plate reader. The E0771 does not compare with the plate reader data; conversely, the 4T07 data show an increasing trend with the irradiated cells consuming more oxygen. This is comparable to the plate reader data. 


\section{Discussion}

\subsection{OCRs Quantified via BNP Emissions are a Viable Alternative to the Seahorse Assay}

The BNPs show consistency in their response to different oxygen environments over time. While the overall intensity might decrease (though not significantly in our experience after tracking BNPs for six months), the ratiometric intensities remain stable; however, in our experience, the BNPs intensity shows a minor dependence on experimental temperature. For this reason, we maintained $37^{\circ} \mathrm{C}$ in this experiment (via the plate-reader). The equivalence between the gold-standard Seahorse assay (via the Bland-Altman analysis) with our nanoparticle method highlights the success of this novel ratiometric OCR measurement technique. The main limitation of the Seahorse assay that we sought to overcome is its inability to translate to more complex systems-either 3D cultures or in vivo. Our successful OCR quantification of two cell lines opens many possibilities for future studies that require dynamic monitoring of OCR of cells. Furthermore, with modifications to the optical imaging setup in the microscope, imaging during OCR measurements should be possible. By outfitting a mouse with a dorsal window chamber, injecting tumor cells, and applying BNPs, we can measure the $\mathrm{pO}_{2}$ across the window using the microscope as described in previous studies. ${ }^{33,38}$ By measuring the local $\mathrm{pO}_{2}$ gradients near vessels and combining these results with theoretical simulations using known oxygen transport parameters, and oxygen diffusivity, we can extrapolate the local OCR across the tumor. This method is similar to the experiment by Dewhirst et $\mathrm{al}^{46}{ }^{46}$ in which they used oxygen electrodes and deduced local OCR in rat dorsal window chamber models.

Like other methods for measuring the OCR, oxygen diffusion through permeable materials was significant despite the addition of an oil barrier; however, the substantial effects of diffusion were controlled through the use of control wells (wells without cells). Once we took this diffusion into account, our method via the plate reader produced repeatable, reliable OCRs.

Though the BNPs, when used with a plate reader, are a sensitive and objective method for quantifying OCR, the main advantage is the ability to extend the method to optical imaging. With the addition of a Quad-View filter system and a MATLAB analysis, we can image and quantify fluorescence and phosphorescence (Fig. 6). Not only does that allow in vitro analyses of cell heterogeneity and morphology, but it is the only method for quantifying the OCR in tumor spheroids, tumor emboli, and ex vivo, live-tissue sections. This is notably different than the Seahorse assay in which specific microplates and cartridges are used to measure the OCR. For heterogenous samples (on slides or in plates) or complex, 3D culture models (that require specialized growing conditions and plates), microscope-based OCR measurements are invaluable. For our system, the microplate is necessary to determine the baseline oxygen percentages due to its straightforward calibration of the BNPs; optical imaging would be a secondary metric for determining relative changes in OCR. The latter is particularly useful because the OCRs may be modulated by extrinsic factors such as environmental hypoxic conditions instead of chemically-induced mitochondrial changes in the cells. Finally, this dual-modality method uses standard technology (plate reader and confocal microscope) found in most labs and; with the exception of the BNPs, there are no expensive cartridges or additives necessary for baseline measurements of OCR.

\subsection{Noisy Microscope Data Diminishes BNP-Measured OCR Sensitivity}

Our data are particularly subject to noise in the microscope with large standard deviations; however, the same trends as shown in the plate reader data are evident (i.e., the irradiated 4T07 cells show a higher OCR than the unirradiated cells). One source of noise that could be more tightly controlled is the depth-of-field; it is well-known that the amount of oxygen in an in vitro system is highly dependent on the depth of the measurement. In an unpublished study from our group, the difference between the $\mathrm{pO}_{2}$ at the surface level of media was compared with the $\mathrm{pO}_{2}$ at the cellular level using an $\mathrm{O}_{2}$ microelectrode. For all four cell lines (Hct1 16, 4T1, 786-0, and $\mathrm{R} 3230 \mathrm{Ac})$, the $\mathrm{pO}_{2}$ at the cellular level was significantly lower $(p<10-6, T$-test $)$ than at the media surface. ${ }^{47}$ In the microscope, the depth at which we are imaging is variable, depending on where the cells are in focus and including significant background media signal (from intensities at various depths). According to our unpublished study, the $\mathrm{pO}_{2}$ will also be 
inaccurate if we are not imaging at a particular depth. Moreover, the analysis averages the signal over the entire field of view instead of just the cells. A masking protocol for areas of interest (i.e., cells) would likely decrease the uncertainty of the measurements significantly. The plate-reader uses a bottom-read technique that eases this noise effect, providing a more robust OCR measurement. By utilizing a structure illumination technique, the signal recorded at the point of focus (at the cells) would afford a measurement that provides an accurate data point at the cell layer.

\subsection{OCRs of Irradiated Cell Lines Show Opposite Trends}

The OCR measurements across the experimental (irradiated) and baseline (unirradiated) cells were consistent. E0771 demonstrates a highly significant reduction in OCR between irradiated and unirradiated cells. Interestingly, though both cell lines were irradiated at their $50 \%$ survival fraction, they demonstrate opposite trends: irradiated E0771 cells exhibit a lower OCR than unirradiated cells, while irradiated 4T07 cells exhibit a higher OCR than unirradiated cells. Processes involved in energy production make up the majority of the oxygen consumption of the basal respiration rate of unirradiated cells, and after irradiation, the observed changes in the OCR of E0771 cells may be indicative of a decrease in the membrane potential required to produce ATP. ${ }^{22,48}$ The observed increase in OCRs in the 4T07 cells may be attributed to increases in nuclear DNA repair; there are multiple studies that demonstrate these mechanistic correlations in irradiated cancer cells. ${ }^{48-50}$ For 4 T07 specifically, there is likely a correlation between nuclear DNA repair and enhancement of cyclin-dependent kinase 1, pyruvate dehydrogenase kinase 1, and glucose metabolism in response to radiation. ${ }^{48-51}$ In a study by Qin et al, ${ }^{48}$ three cell lines (MCF10A, HK18, and P + JB6), exhibited a three-fold increase in OCR (measured via a Clarke-type oxygen electrode) over the course of $40 \mathrm{~h}$ after a single dose of ionizing radiation. This increase could be the impact of DNA repair, an ATP-consuming process. ${ }^{48}$ While this study, among others, ${ }^{50}$ indicates that the majority of cancerous cell lines demonstrate an increased OCR after radiation exposure, E0771 demonstrates a different trend. For the E0771 cells, we do not see increased OCR post-radiation exposure. E0771 cells have a lower basal OCR and a higher sensitivity to radiation than $4 \mathrm{~T} 07$, both notable effects of a decreased energy metabolism. ${ }^{49}$ There could be a number of competing effects in these results: depending on the fraction of cells that died via apoptosis and double-strand DNA breaks, the necessity of increasing the ATP consumption to mitigate damage will be smaller. The change from anaerobic metabolism to aerobic metabolism would also modulate oxygen metabolism, depending on factors such as hypoxia-inducible factor 1alpha and other modulators of glucose metabolism. These are only a few of the possibilities that explain the data, but it is clear that a dedicated experiment aimed toward defining the underlying mechanisms for the differential effect of radiation on the OCR of these two cells lines would be required.

\section{Conclusion}

Our data demonstrate a novel method for precisely and accurately quantifying the OCRs of cell in vitro via ratiometric oxygen sensing. Our results show comparable values relative to the standard Seahorse assay; however, unlike the Seahorse assay, this method can be extended to in vivo studies in which fluorescence imaging is available and optical microscopy imaging. While this method has yet to be tested on human-derived cancer cell lines or in conjunction with chemicals that alter the cellular metabolism, there is a wide field in which BNP-based OCR measurements are effective and advantageous.

\section{Disclosures}

The authors have no conflicts of interest to disclose.

\section{Acknowledgments}

We thank the National Institutes of Health (Grant No. R01 CA167250) and UVA Cancer Center (Grant No. P30 CA44579) for support of this work. We utilized the Duke Optical Molecular 
Imaging and Analysis shared resource at Duke, which is supported by the Duke Cancer Institute and Duke School of Medicine.

\section{References}

1. L. D. Osellame, T. S. Blacker, and M. R. Duchen, "Cellular and molecular mechanisms of mitochondrial function," Best Pract. Res. Clin. Endocrinol. Metab. 26(6), 711-723 (2012).

2. J. E. Biaglow et al., "MIBG inhibits respiration: potential for radio- and hyperthermic sensitization," Int. J. Radiat. Oncol. Biol. Phys. 42(4), 871-876 (1998).

3. C. Diepart et al., "Arsenic trioxide treatment decreases the oxygen consumption rate of tumor cells and radiosensitizes solid tumors," Cancer Res. 72(2), 482-490 (2012).

4. H. Pelicano et al., "Inhibition of mitochondrial respiration: a novel strategy to enhance druginduced apoptosis in human leukemia cells by a reactive oxygen species-mediated mechanism," J. Biol. Chem. 278(39), 37832-37839 (2003).

5. B. Brunmair et al., "Thiazolidinediones, like metformin, inhibit respiratory complex I: a common mechanism contributing to their antidiabetic actions?" Diabetes 53(4), 1052-1059 (2004).

6. T. W. Secomb, R. Hsu, and M. W. Dewhirst, "Synergistic effects of hyperoxic gas breathing and reduced oxygen consumption on tumor oxygenation: a theoretical model," Int. J. Radiat. Oncol. Biol. Phys. 59(2), 572-578 (2004).

7. P. Vaupel, "Tumor microenvironmental physiology and its implications for radiation oncology," Semin. Radiat. Oncol. 14(3), 198-206 (2004).

8. S. Zhou et al., "Frequency and phenotypic implications of mitochondrial DNA mutations in human squamous cell cancers of the head and neck," Proc. Natl. Acad. Sci. U.S.A. 104(18), 7540-7545 (2007).

9. J. A. Petros et al., "mtDNA mutations increase tumorigenicity in prostate cancer," Proc. Natl. Acad. Sci. U.S.A. 102(3), 719-724 (2005).

10. Y. Chen et al., "Oxygen consumption can regulate the growth of tumors, a new perspective on the Warburg effect," PLoS One 4(9), e7033 (2009).

11. T. W. Secomb et al., "Analysis of the effects of oxygen supply and demand on hypoxic fraction in tumors," Acta Oncol. 34(3), 313-316 (1995).

12. T. W. Secomb et al., "Theoretical simulation of oxygen transport to tumors by threedimensional networks of microvessels," Adv. Exp. Med. Biol. 454, 629-634 (1998).

13. M. Riester et al., "The Warburg effect: persistence of stem-cell metabolism in cancers as a failure of differentiation," Ann. Oncol. 29(1), 264-270 (2018).

14. P. Vaupel and G. Thews, " $\mathrm{PO}_{2}$ distribution in tumor tissue of DS-carcinosarcoma," Oncology 30(6), 475-484 (1974).

15. M. L. Karnovsky and J. A. Badwey, "[17] Respiratory burst during phagocytosis: an overview," Methods Enzymol. 132, 353-354 (1986).

16. F. Kallinowski et al., "Growth-related changes of oxygen consumption rates of tumor cells grown in vitro and in vivo," J. Cell. Physiol. 138(1), 183-191 (1989).

17. O. K. Glass et al., "Differential response to exercise in claudin-low breast cancer," Oncotarget 8(60), 100989 (2017).

18. C. G. Perry et al., "Methods for assessing mitochondrial function in diabetes," Diabetes 62(4), 1041-1053 (2013).

19. B. Wang and P. R. Ogilby, "Activation barriers for oxygen diffusion in polystyrene and polycarbonate glasses: effects of codissolved argon, helium, and nitrogen," Can. J. Chem. 73(11), 1831-1840 (1995).

20. L. Poulsen et al., "Oxygen diffusion in bilayer polymer films," J. Phys. Chem. B 107(50), 13885-13891 (2003).

21. A. A. Gerencser et al., "Quantitative microplate-based respirometry with correction for oxygen diffusion," Anal. Chem. 81(16), 6868-6878 (2009).

22. A. S. Divakaruni et al., "Chapter sixteen-analysis and interpretation of microplate-based oxygen consumption and pH data," Methods Enzymol. 547, 309-354 (2014).

23. C. C. Cook et al., "Consumption of oxygen: a mitochondrial-generated progression signal of advanced cancer," Cell Death Dis. 3, e258 (2012). 
Rickard et al.: Dual-emissive, oxygen-sensing boron nanoparticles quantify oxygen consumption rate...

24. S. Anoopkumar-Dukie et al., "Mitochondrial modulation of oxygen-dependent radiosensitivity in some human tumour cell lines," Br. J. Radiol. 82(982), 847-854 (2009).

25. N. Nishida et al., "3-methyl pyruvate enhances radiosensitivity through increasing mitochondria-derived reactive oxygen species in tumor cell lines," J. Radiat. Res. 55(3), 455-463 (2014).

26. M. Benej et al., "Papaverine and its derivatives radiosensitize solid tumors by inhibiting mitochondrial metabolism," Proc. Natl. Acad. Sci. U.S.A. 115(42), 10756-10761 (2018).

27. B. C. Dickinson, C. Huynh, and C. J. Chang, "A palette of fluorescent probes with varying emission colors for imaging hydrogen peroxide signaling in living cells," J. Am. Chem. Soc. 132(16), 5906-5915 (2010).

28. B. C. Dickinson, D. Srikun, and C. J. Chang, "Mitochondrial-targeted fluorescent probes for reactive oxygen species," Curr. Opin. Chem. Biol. 14(1), 50-56 (2010).

29. A. J. Meyer and T. P. Dick, "Fluorescent protein-based redox probes," Antioxid. Redox. Signal 13(5), 621-650 (2010).

30. L. P. Roma et al., "Mechanisms and applications of redox-sensitive green fluorescent protein-based hydrogen peroxide probes," Antioxid. Redox Signal 29(6), 552-568 (2018).

31. P.-Z. Chen et al., "Difluoroboron $\beta$-diketonate dyes: spectroscopic properties and applications," Coordin. Chem. Rev. 350, 196-216 (2017).

32. G. Zhang et al., "A dual-emissive-materials design concept enables tumour hypoxia imaging," Nat. Mater. 8(9), 747-751 (2009).

33. G. M. Palmer et al., "In vivo optical molecular imaging and analysis in mice using dorsal window chamber models applied to hypoxia, vasculature and fluorescent reporters," Nat. Protoc. 6(9), 1355-1366 (2011).

34. P. Lehner et al., "Ultra-sensitive optical oxygen sensors for characterization of nearly anoxic systems," Nat. Commun. 5, 4460 (2014).

35. J. X. Wang et al., "Ratiometric $\mathrm{O}_{2}$ sensing based on selective self-sensitized photooxidation of donor-acceptor fluorophores," Chem. Commun. 55(49), 7017-7020 (2019).

36. F. R. Kersey et al., "Stereocomplexed poly(lactic acid)-poly(ethylene glycol) nanoparticles with dual-emissive boron dyes for tumor accumulation," ACS Nano 4(9), 4989-4996 (2010).

37. C. Kerr et al., "Luminescent difluoroboron beta-diketonate PLA-PEG nanoparticle," Biomacromolecules 18(2), 551-561 (2017).

38. C. A. DeRosa et al., "Oxygen sensing difluoroboron beta-diketonate polylactide materials with tunable dynamic ranges for wound imaging," ACS Sens. 1(11), 1366-1373 (2016).

39. C. A. DeRosa et al., "Oxygen Sensing difluoroboron dinaphthoylmethane polylactide," Macromolecules 48(9), 2967-2977 (2015).

40. A. Carreau et al., "Why is the partial oxygen pressure of human tissues a crucial parameter? Small molecules and hypoxia," J. Cell. Mol. Med. 15(6), 1239-1253 (2011).

41. L. A. Kinard, F. K. Kasper, and A. G. Mikos, "Synthesis of oligo(poly(ethylene glycol) fumarate)," Nat. Protoc. 7(6), 1219-1227 (2012).

42. M. W. Irvine et al., "Piperazine-2,3-dicarboxylic acid derivatives as dual antagonists of NMDA and GluK1-containing kainate receptors," J. Med. Chem. 55(1), 327-341 (2012).

43. D. B. Williams and M. Lawton, "Drying of organic solvents: quantitative evaluation of the efficiency of several desiccants," J. Org. Chem. 75(24), 8351-8354 (2010).

44. H. Z. Jin et al., "Dendron-jacketed electrophosphorescent copolymers: improved efficiency and tunable emission color by partial energy transfer," Macromolecules 44(24), 9556-9564 (2011).

45. R. Bro, "The N-way toolbox," MATLAB Central File Exchange (2020).

46. M. W. Dewhirst et al., "Determination of local oxygen consumption rates in tumors," Cancer Res. 54(13), 3333-3336 (1994).

47. L. I. Cárdenas-Navia, Characterization and Modeling of Fluctuating Hypoxia in Breast Cancer, p. 148, Duke University (2008).

48. L. Qin et al., "CDK1 enhances mitochondrial bioenergetics for radiation-induced DNA repair," Cell Rep. 13(10), 2056-2063 (2015).

49. H. Shen et al., "Sensitization of glioblastoma cells to irradiation by modulating the glucose metabolism," Mol. Cancer Ther. 14(8), 1794-1804 (2015). 
Rickard et al.: Dual-emissive, oxygen-sensing boron nanoparticles quantify oxygen consumption rate...

50. K. Yamamoto et al., "Evaluation of mitochondrial redox status and energy metabolism of X-irradiated HeLa cells by LC/UV, LC/MS/MS and ESR,” Free Radic. Res. 52(6), 648-660 (2018).

51. T. Liu and H. Yin, "PDK1 promotes tumor cell proliferation and migration by enhancing the Warburg effect in non-small cell lung cancer," Oncol. Rep. 37(1), 193-200 (2017).

Ashlyn G. Rickard is a PhD candidate at Duke University in the program of medical physics. She received her BS degree in physics from the University of North Carolina at Asheville and her MS degree in medical physics from Duke University. Her current research interests include methods to measure and treat hypoxia in a radiation oncology setting. This includes fluorescent microscopy in murine dorsal window chamber models to investigate local hypoxia and electron paramagnetic oxygen imaging to quantify the effects of radiosensitizers.

Biographies of the other authors are not available. 